\title{
A Technical Note, Looback Options: a comparison between Monte Carlo Techniques
}

\author{
Marcelo González A. \\ Facultad de Economía y Negocios \\ Universidad de Chile \\ mgonzale@unegocios.cl
}

\author{
Antonino Parisi F. \\ Facultad de Economía y Negocios \\ Universidad de Chile \\ aparisi@unegocios.cl \\ Arturo Rodríguez P. \\ Facultad de Economía y Negocios \\ Universidad de Chile \\ arodriguez@unegocios.cl
}

\begin{abstract}
Looback options are path dependent contingent claims whose payoffs depend on the extrema of the underlying asset price over a certain time interval. In this note we compare the performance of two Monte Carlo techniques to price lookback options, a crude Monte Carlo estimator and Antithetic variate estimator. We find that the Antithetic estimator performs better under a variety of performance measures.
\end{abstract}

Keywords: Monte Carlo, Simulations, Options. 
Resumen

Las opciones Looback son contratos que exhiben dependencia de sendero y cuyo ingreso bruto depende de los valores extremos del activo subyacente en un intervalo de tiempo determinado. En esta nota comparamos el desempeño de dos técnicas de Monte Carlo: estimador directo de Monte Carlo y estimador de variables antitético. Nuestros resultados muestran que el estimador antitético ofrece un mejor desempeño de acuerdo a distintas medidas de desempeño.

Palabras clave: Monte Carlo, simulaciones, opciones.

\section{Introduction}

Options on stocks were first traded on an organized exchange in 1973. Since then, there has been a dramatic growth in option markets. Options are now traded on many different exchanges throughout the world. Huge volumes of options are also traded over the counter by banks and other financial institutions. The underlying assets include stocks, stock indices, foreign currencies and debt instruments.

Part of this growth in the derivatives market it is due to the increasing importance of non-standard option contracts, that is, options that are not a vanilla put or call. These kinds of contracts have been developed by banks and financial institutions at an increasing rate to meet the needs of their clients. An important class within these contracts is path dependent options. A path dependent option is an option whose payoff at exercise or expiry depends, in some nontrivial way, on the past history of the underlying asset price as well as its spot price at exercise or expiry.

Lookback options are an example of this kind of financial instruments. They are path dependent options whose payoffs depend on the maximum or minimum attained over a certain period of time by the security's price. For example, a European lookback put gives 
the right to the holder to sell a stock at a certain date in the future for a price equal to the maximum price of the stock during the life of the option. On the other hand, a standard European lookback call is the right to buy at a historical lowest price over a certain period. These kinds of options look like a plain vanilla put or call with the terminal security price replaced by either the maximum or the minimum price during the life of the contract. Such options give the holder an extremely advantageous payoff. Using lookback options, one can design a strategy enabling the investor to buy at the low and sell at the high. They are therefore relatively expensive. Goldman, Sossin and Gatto (1979) note that although in a perfect market such options would not expand the investment opportunity set in a real market they may present some peculiar attractive features such as the minimization of regret and maybe more importantly the opportunity to take advantage of either special information or skill on behalf of the trader.

Work on standard lookback call options was first done by Goldman et al. (1979), Goldman, Sosin, and Shepp (1979), and Conze and Viswanathan (1991). All of the above studies show that in the Black-Scholes framework it is possible to obtain closed form solutions for the value of European lookback options. This framework assumes that the underlying asset follows a geometric Brownian motion which in turn implies that the underlying asset is lognormally distributed.

The purpose of this technical note is to implement two Monte Carlo techniques to obtain a numerical solution to the problem of pricing a Lookback option. We choose the Black-Scholes framework so that we can compare both techniques with the theoretical price. The techniques proposed are simple and flexible, though, in the sense that can be easily modified to accommodate different process for the underlying stock price. We use three alternative measures to compare the performance of these techniques. Section 2 of this note provides a brief description of option pricing and motivates the use of Monte Carlo methods in this context. Section 3 discusses the 
methodology. Section 4 analyzes results. Section 5 gives the conclusions and discusses further applications of the method

\section{Option Pricing: a Review}

The price of a stock option is a function of the underlying stock's price and time. Therefore, virtually every option pricing model starts by postulating a process for the stock price dynamics. Two reasonable assumptions regarding the process are that both the variance and the expected return in a short period of time are independent of the stock price. In other words, an investor is just as uncertain as to his percentage return when the stock price is $\$ 100$ as when it is $\$ 10$. A model that captures these characteristics and that it is mathematically tractable is the Geometric Brownian Motion. Which can written as

$$
d s=u s d t+\sigma s d z \text { where } d z=\varepsilon \sqrt{d t} \quad \varepsilon \sim N(0,1)
$$

Here $d s$ denotes the change in the stock price in the next infinitesimal moment. Also, the first term of the equation represents the expected return or drift of the process and the second term is the stochastic component reflecting the uncertainty about future prices. The next steps to price an option following the PDE approach follow:

1) Obtain the dynamic equation for the option price (This is done by applying Ito's Lemma from Stochastic Calculus). The resulting equation is structurally similar to the equation for the stock prices and shares the same stochastic term.

2) Combine both equations in a way that the stochastic term disappears. The resulting portfolio of a stock and an option is therefore riskless and its price should be equal to the price of riskless instrument. 
3) The solution to the PDE derived in 2) is the price of the option.

For the purpose of this note, the important thing to notice is that the equation in derived in the second step does not contain the (unobservable) required return on the stock, which leads to the so called risk neutral approach to option valuation. This approach can be implemented in the following way:

- Replace the drift in the process with the risk-free rate (observable).

- $\quad$ Calculate the expected payoff using the distribution implied by the new process.

- $\quad$ Discount the resulting value at the risk-free rate.

For the lookback case the risk neutral valuation formula can be written as:

$$
L=E\left[\max _{0<t<T} p(t)-p(T)\right] e^{-r T}
$$

where $L$ denotes the lookback price and $p(t)$ is the stock price at time $t$. Note that under the new process the distribution of future prices is lognormal with an expected value given by:

$$
E\left(p_{t}\right)=p_{0} e^{r t}
$$

where $r$ is the risk-free rate and is distributed standard normal.

The objective is to estimate the equation for $L$ using the Monte Carlo method. 


\section{Methodology}

The two techniques implemented in this note are the Crude Monte Carlo and the Antithetic Variates. To set up the Crude Monte Carlo, we take the following steps:

- Simulate a path for future prices according to the lognormal distribution.

- $\quad$ Choose a maximum price from the path generated.

- $\quad$ Letting $\mathrm{m}=$ number of iterations, the Crude Monte Carlo estimator is given by:

$$
L=e^{-r T} 1 / m \sum_{j=1}^{m} y_{j n}-P(0) \quad y_{j n}=\max _{o<k<n} P_{j k} .
$$

To obtain the Antithetic Variates estimator, for each simulated path, another is generated by reversing the sign of each of the IID standard normal on which the price path is based. The Antithetic estimator is given by:

$$
L=e^{-r t} 1 / 2 m\left(\sum_{j=1}^{m} y_{j n}^{1}+\sum_{j=1}^{m} y_{j n}^{2}\right)-P(0) .
$$

Note that the second estimator doubles the number of iterations by generating a second sample based on the first one. Further, the second sample is negatively correlated with the first one. Because of those two characteristics the antithetic estimator variance should be smaller than the crude.

To compare the performance of both estimators, we use three alternative measures:

- $\quad$ Efficiency Measure. $n c_{\sigma c}^{2} / n a_{\sigma a}^{2}$ where $n c$ and $n a$ denote the CPU time of the Crude and Antithetic estimators, 
respectively. A ratio greater that 1 would indicate that the Antithetic estimator is more efficient.

- $\quad$ Accuracy Measure. Standard error of the estimate, since this measure is inversely proportional to the number of iterations, we expect a priori that the Antithetic estimator would be more accurate.

- Deviation from theoretical value. Since in the particular case of the Lookback option there is close-form solution, we also compare the estimated values with the formula values.

\section{Results}

A comparison of the Crude Monte Carlo to the Antithetic estimator is provided in table 1. M denotes the number of iterations. The parameter values for $\mathrm{r}$ and sigma are set equal to $5 \%$ and $20 \%$, respectively. $N$ denotes sample size, which is the number of prices generated in each iteration. $\mathrm{Pc}, \mathrm{Pa}$ and $\mathrm{Pf}$ are the Crude Monte Carlo, the Antithetic and the formula prices. For all the simulations, the ratio of the standard error of Antithetic estimator (SEa) to the standard error of the Crude Monte Carlo (SEc) is around $0.035 / 0.086=0.407$, a reduction of about $60 \%$. In comparison, a doubling of the number of replications from $m$ to $2 m$ for the Crude Monte Carlo estimator would yield a ratio of $1 / \sqrt{2}=0.707$, only a $29 \%$ reduction. Therefore, the negative correlation induced by the sampling procedure accounts for a good portion of the reduction in variance. Now, since the generation of the second sample does not require an additional simulation it is not surprising that the Antithetic method turns out to be more efficient.

The difference between the estimated prices and the formula price arises from two sources: sampling variation and the discreteness of the simulated prices. The former source of discrepancy is controlled by the number of iterations, while the latter source is controlled by the number of prices simulated in each 
replication. A comparison between tables I and II below shows that for this experiment the number simulated prices plays a more important role. Note also that the Crude estimator attains the minimum difference when $\mathrm{n}=2000$ and $\mathrm{m}=5000$. According to the deviation measure both estimators systematically underprice the lookback option. This is to be expected because we are selecting a maximum over $\mathrm{k}$ prices, where $\mathrm{k}$ goes from 0 to $\mathrm{n}$, as $\mathrm{n}$ increases the maximum is likely to increase as well. Therefore, the formula price, which is based on a continuos time price, will almost always exceed the simulation price, which is discretized.

Table 1

$(\mathrm{m}=5000, \mathrm{r}=0.05$, sigma $=0.20, \mathrm{Pf}=5.71)$

\begin{tabular}{c|ccccccc}
\hline $\mathrm{N}$ & $\mathrm{Pc}$ & $\mathrm{Pa}$ & $\mathrm{Sec}$ & $\mathrm{SEa}$ & Effic. & Pf-Pc & Pf-Pa \\
\hline 250 & 5.279 & 5.409 & 0.086 & 0.035 & 3.738 & 0.431 & 0.301 \\
500 & 5.612 & 5.506 & 0.088 & 0.037 & 5.921 & 0.098 & 0.204 \\
1000 & 5.443 & 5.536 & 0.086 & 0.036 & 7.334 & 0.267 & 0.174 \\
2000 & 5.653 & 5.570 & 0.086 & 0.035 & 6.021 & 0.057 & 0.140 \\
5000 & 5.629 & 5.632 & 0.089 & 0.037 & 2.907 & 0.081 & 0.078 \\
\hline
\end{tabular}

\section{Table 2}

\begin{tabular}{c|ccccccc}
\multicolumn{8}{c}{$(\mathrm{m}=10000, \mathrm{r}=0.05$, sigma $=0.20)$} \\
\hline $\mathrm{N}$ & Pc & Pa & Sec & SEa & Effic. & Pf-Pc & Pf-Pa \\
\hline 250 & 5.364 & 5.343 & 0.060 & 0.025 & 6.055 & 0.346 & 0.367 \\
500 & 5.519 & 5.463 & 0.062 & 0.025 & 5.950 & 0.191 & 0.247 \\
1000 & 5.606 & 5.581 & 0.062 & 0.026 & 6.044 & 0.104 & 0.129 \\
2000 & 5.618 & 5.624 & 0.062 & 0.025 & 5.494 & 0.092 & 0.086 \\
5000 & 5.553 & 5.623 & 0.060 & 0.025 & 5.012 & 0.157 & 0.087 \\
\hline
\end{tabular}

However, this observation does not imply that the Monte Carlo estimators are inferior to the closed-form solution, since real prices are discrete not continuos. Therefore, the difference simply highlights the importance of discretization in the pricing of options. 


\section{Conclusion and Extensions}

The Monte Carlo method represents an alternative to the numerical methods employed in Option pricing. One advantage of the method is that it is very flexible with regard to the distribution of stock prices. Changing the underlying distribution merely involves using a different process for generating the random variates employ in the method. Furthermore, the method is perhaps unique in the sense that the distribution used to generate prices need not have a closed- form analytic expression. This opens the possibility of using empirical distribution to value options. An area worth exploring since the empirical evidence on stock prices distributions is not conclusive ${ }^{1}$. Furthermore, the method also provides measures of accuracy. However, the advantages do not come without price. Some limitations to this approach should be emphasized. The direct Monte Carlo approach presented here would require a "Monte Carlo on Monte Carlo" algorithm to be applied to American options. Consider a simulated path and a point $(\mathrm{P}(\mathrm{t}), \mathrm{t})$ on that path. To value an American option one must decide if it is optimal to exercise at that point. This in turn requires evaluation of the risk neutral formula and therefore requires continuation from $(\mathrm{P}(\mathrm{t}), \mathrm{t})$ on many paths. Thus this direct Monte Carlo simulation of the American options requires a set of continuously branching paths, which is computationally intractable. Several recent methods have been proposed to deal with theses difficulties, though. For example, Longstaff and Schwartz (2001) and Tsitsiklis and Van Roy (1999), introduced a new approach to Monte Carlo valuation by replacing the future expectation required by the risk neutral approach by a least squares interpolation. The method has been shown to work well on a good selection of examples, but it still needs validation for more complicated examples, such as American Asian options with a

\footnotetext{
${ }^{1}$ See for example Carr et al. (2002) or Login (1996).
} 
moving window over which the average is taken. In contrast, Rogers (2002) propose an approach which makes no attempt to approximate an exercise policy, but instead gives an upper bound to the true price by means of Martingale optimization. The main difficulty with this approach lies in the method for choosing the Martingale in order to get a good approximation.

\section{References}

Carr, P., H. Geman, D. Madan and M. Yor (2002), "The fine structure of asset returns: an empirical investigation", Journal of Business, 75, pp. 305-323.

Conze A., and Viswanathan (1991), "Path dependent options: The Case of Lookback Options", Journal of Finance, 46, pp. 1893-1906.

Goldman, B.M., H.B. Sosin, and M.A. Gatto (1979), "Path dependent options: "Buy at the low, sell at the high"”, Journal of Finance, 34, pp. 1111-1127.

Goldman, B.M., H.B. Sosin and L.A. ShePp (1979), "On contingent claim that insures ex-post optimal stock market timing", Journal of Finance, 34, pp. 401-414.

LOGIN F. (1996), "The asymptotic distribution of extreme stock market returns", Journal of Business, 60, pp. 383-408.

LongstafF F., and E. Schwartz (2001), "Valuing American Options by simulation: A Simple Least Squares Approach", The Review of Financial Studies, 14, pp. 113-147.

Rogers L.C.G. (2002), "Monte Carlo Valuation of American Options", Mathematical Finance, 12, pp. 271-286, Vol. 29, pp. 449-470. 